\title{
TUMOR PARDO MULTIFOCAL COMO MANIFESTACIÓN DEL HIPERPARATIROIDISMO PRIMARIO POR ADENOMA PARATIROIDEO ASOCIADO A CARCINOMA PAPILAR DE TIROIDES*
}

\author{
Drs. Williams Arias G. ${ }^{1}$, Andrés Ayala O. ${ }^{2}$, Felipe Pacheco B. ${ }^{3}$, David Barzallo S. ${ }^{2}$ \\ 1 Servicio de Cirugía, Hospital Quito $N^{o} 1$ de la Policía Nacional. \\ 2 Residente de Cirugía General. Universidad Internacional del Ecuador. Hospital Metropolitano. \\ 3 Servicio de Cirugía, Hospital Metropolitano. \\ Quito-Ecuador.
}

\begin{abstract}
Brown tumor of the pelvis associated with hyperparathyroidism

Background: Brown tumors of bones are an uncommon manifestation of hyperparathyroidism. Case report: We report a 35 years old male presenting with pain and paresis of the left superior limb. Part of his humerus was excised due to a diagnosis of a giant cell tumor. He was admitted again to the hospital due to pelvic pain, malaise and constipation. A right cervical nodule was found. Laboratory evaluation confirmed the presence of a hyperparathyroidism. The biopsy of the pelvic lesion disclosed a brown tumor. The patient was subjected to a parathyroidectomy and the pathological study of the surgical piece showed a right parathyroid adenoma and a right thyroid papillary micro carcinoma. In the postoperative period the patient had a hungry bone syndrome, which was adequately treated.
\end{abstract}

Key words: Hyperparathyroidism, brown tumor, parathyroidectomy.

\section{Resumen}

Introducción: La paratohormona es una hormona encargada de la homeostasis del calcio, el hiperparatiroidismo es una patología con manifestaciones renales y óseas, el Tumor Pardo es una rara presentación de esta enfermedad. Caso clínico: Hombre de 35 años con dolor y paresia en extremidad superior izquierda, fue resecado parte del húmero por un diagnóstico de Tumor de Células Gigantes; reingresa con dolor pélvico derecho, malestar general, astenia y estreñimiento. Se descubre un nódulo cervical derecho e hipersensibilidad en la pelvis derecha. Los exámenes de laboratorio muestran hiperparatiroidismo; la biopsia de la lesión pélvica es diagnóstica de Tumor Pardo, encontrándose además una hipercaptación paratiroidea derecha. Operado,

\footnotetext{
*Recibido el 13 de marzo de 2014 y aceptado para publicación el 29 de abril de 2014.
}

Los autores no refieren conflictos de interés.

Correspondencia: Dr. Williams Arias G. willariasmx@yahoo.com.mx 
el diagnóstico histopatológico fue: Adenoma paratiroideo derecho y un micro carcinoma papilar tiroideo; en el post-operatorio desarrolló un Síndrome de Bone Hunger, el cual fue superado y dado de alta. Discusión y conclusiones: El Tumor Pardo no es una verdadera neoplasia; producido por intensa actividad osteoclástica, tiene características histológicas y radiológicas inespecíficas y su diagnóstico se realiza por datos clínicos y bioquímicos. El hiperparatiroidismo puede llevar a la formación de Tumores Pardos; se sugiere realizar estudios de la glándula tiroides en pacientes con hiperparatiroidismo.

Palabras clave: Hipercalcemia, hiperparatiroidismo, tumor pardo, micro-carcinoma.

\section{Introducción}

La paratohormona es una hormona proteica secretada por las glándulas paratiroides cuya principal función es la regulación de la homeostasis del calcio, su sobreproducción a partir de una o varias glándulas produce hipercalcemia; este estado de hiperparatiroidismo es la tercera endocrinopatía más común y afecta al $0,3 \%$ de la población en general y a 1-3\% de las mujeres posmenopáusicas ${ }^{1,2}$.

En EEUU la incidencia de hiperparatiroidismo ha ido en aumento, esto debido a la modernización de técnicas de dosificación sanguínea de calcio sérico, a cerca de 22 casos por 100.000 personas por año ${ }^{1,3}$. En el 85 a 95\% de los casos el hiperparatiroidismo es producido por un adenoma paratiroideo, mientras que el $5-10 \%$ es producido por enfermedad multi-glandular y en menos del $1 \%$ por el carcinoma paratiroideo ${ }^{4}$.

Los principales efectos del hiperparatiroidismo se manifiestan a nivel del sistema renal y óseo ${ }^{5}$. Las manifestaciones esqueléticas son raras, sin embargo, es común la disminución de la densidad mineral de los huesos corticales ${ }^{6}$; una rara complicación de presentación tardía es el Tumor Pardo que se caracteriza por un intenso proceso reactivo del tejido óseo secundario a la rápida acción osteoclástica de la hipercalcemia ${ }^{7}$, más raro aún es su asociación con un Carcinoma Papilar tiroideo que fue descrito en 1935 por Hellwig, quien notó la presencia de nódulos tiroideos en ratas que recibieron una dieta rica en calcio y pobre en yodo ${ }^{8}$; más tarde, en 1954 , Taylor reportó la capacidad bociógena del calcio en ratas y la posible relación de la hipercalcemia para el desarrollo de patología tiroidea maligna9 .

El presente caso clínico documenta la asociación de un adenoma, que simula un Carcinoma paratiroideo, con un Tumor Pardo multifocal y un Microcarcinoma Papilar de tiroides.

\section{Caso clínico}

Paciente masculino de 35 años con historia de 10 meses de evolución caracterizado por dolor y disminución de la fuerza en hombro y brazo izquierdos, inicialmente atendido por el Servicio de
Traumatología y Ortopedia, quienes documentan la presencia de un tumor en el húmero izquierdo, cuya biopsia diagnostica un Tumor de Células Gigantes, por lo cual se realizó la resección de los dos tercios proximales del húmero y colocación de prótesis. Cuatro meses después el paciente reingresa con dificultad para la marcha por importante dolor en la pelvis derecha, acompañado de malestar general, astenia y estreñimiento. En el examen físico se descubre un nódulo cervical anterior derecho de aproximadamente $3 \mathrm{~cm} \times 2 \mathrm{~cm} \mathrm{x}$ $3 \mathrm{~cm}$, no móvil, de bordes mal definidos, sin dolor ni invasión a piel; además de hipersensibilidad a nivel de la región sacro ilíaca derecha. Los exámenes de laboratorio muestran: Fosfatasa alcalina de $2.700 \mathrm{UI} / \mathrm{L}$, GGT $285 \mathrm{UI} / \mathrm{L}$, Creatinina $0,81 \mathrm{mg} / \mathrm{dl}$, Calcio total $17 \mathrm{mg} / \mathrm{dl}$, Fósforo $2,2 \mathrm{mg} / \mathrm{dl}$, Calcio urinario 528,3 mg/24 h, Fósforo urinario 1,44 g/24 $\mathrm{h}$, hormonas tiroideas normales y Paratohormona $2.802 \mathrm{pg} / \mathrm{ml}$. Con esos hallazgos se procedió a realizar una Gammagrafía ósea con $\mathrm{Tc}^{99}$ que encuentra una hipercaptación a nivel de la articulación sacro ilíaca derecha (Figura 1). Densitometría ósea describe osteoporosis con alto riesgo para fracturas y captación generalizada severamente baja para la edad.

La Resonancia Magnética Nuclear evidenció una lesión de densidad mixta, septada, expansiva del ala ilíaca y del techo acetabular derechos (Figura 2); ante la sospecha de metástasis óseas originadas de un Carcinoma Paratiroideo se realizó una biopsia del tumor ilíaco que muestra numerosas células gigantes tipo osteoclásticas con zonas de hemosiderina y estroma fibroso (Figura 3), hallazgos que sugieren Tumor Pardo. Adicionalmente un estudio de Sestamibi encontró una zona de hipercaptación en el área paratiroidea derecha (Figura 4).

El paciente fue tratado quirúrgicamente, mediante cervicotomía; se encontró un tumor paratiroideo de $3 \times 3 \times 2 \mathrm{~cm}$ firmemente adherido al lóbulo tiroideo derecho y el músculo constrictor inferior de la faringe; además se encontró un nódulo tiroideo palpable de $0,5 \mathrm{~cm}$ de diámetro en lóbulo superior derecho. Se realizó una resección multi estructural que incluye el tumor paratiroideo, músculo constrictor y lóbulo tiroideo derecho, (Figura 5) las demás glándulas paratiroideas fueron de características 


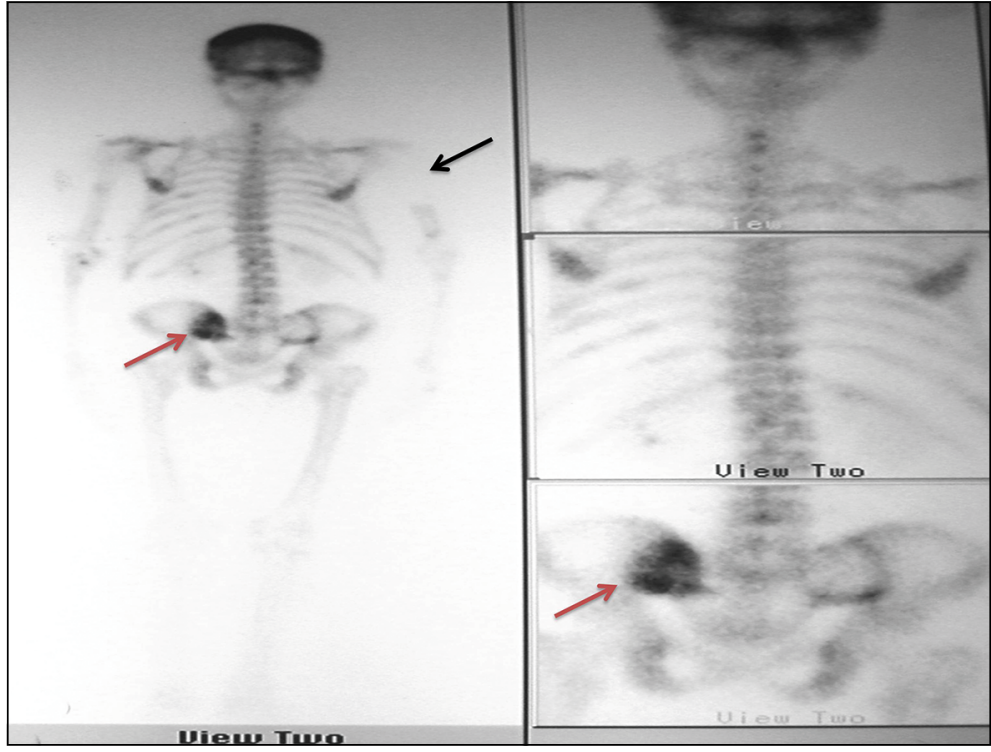

Figura 1. Gammagrafía ósea que muestra hipercaptación a nivel de la articulación sacro ilíaca derecha (Flecha roja) y ausencia de captación a nivel de húmero izquierdo por presencia de prótesis (Flecha negra).

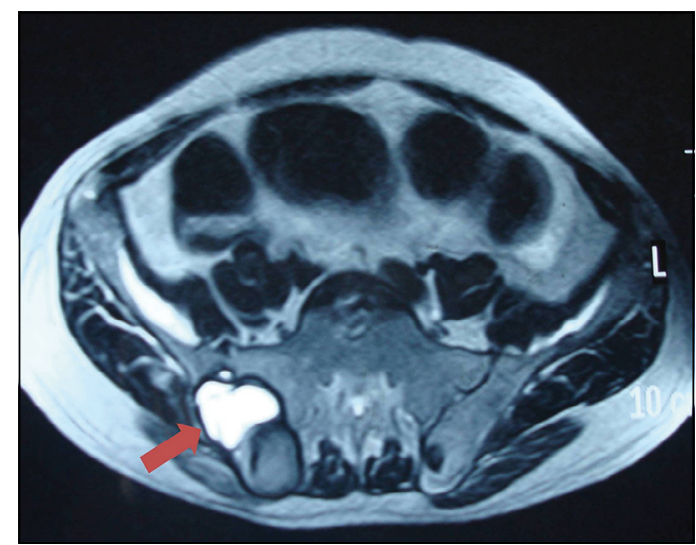

Figura 2. Resonancia Magnética Nuclear que evidencia lesión expansiva de densidad mixta, en el ala ilíaca y el techo acetabular derechos (Flecha roja).

macroscópicas normales. El reporte de histopatología documenta un Adenoma paratiroideo derecho y un micro carcinoma papilar de tiroides (Figura 6). El control de Paratohormona a las 24 h de la cirugía, fue de $125 \mathrm{pg} / \mathrm{ml}$ y Calcio el iónico de $1,17 \mathrm{mg} / \mathrm{dl}$; en los subsiguientes días el paciente desarrolló el Síndrome de Bone Hunger con hipocalcemia sintomática que conllevó a instaurar tratamiento con altas dosis de Gluconato de Calcio, Carbonato de Calcio y Calcitriol. El paciente es dado de alta a los 15 días de hospitalización con niveles normales de Calcio y Paratohormona. Como parte de su seguimiento, después de 3 meses, se realizó una Tomografía

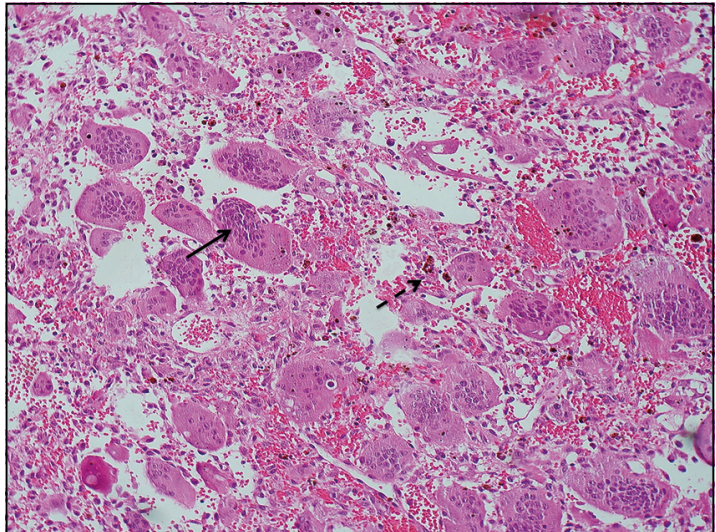

Figura 3. Tumor pardo formado por múltiples depósitos de osteoclastos (Flecha negra) y hemosiderina (Flecha negra entrecortada) (10X).

Computarizada ósea de pelvis en donde se aprecia importante regresión de lesión pélvica.

\section{Discusión}

El hiperparatiroidismo se caracteriza por el aumento de la actividad de las glándulas paratiroideas debido a una alteración intrínseca en la excreción de la hormona paratiroidea (hiperparatiroidismo primario o terciario) o de una alteración extrínseca (hiperparatiroidismo secundario) ${ }^{10}$, que dan como resultado niveles elevados de la hormona paratiroidea que producen hipercalcemia ${ }^{11}$. 


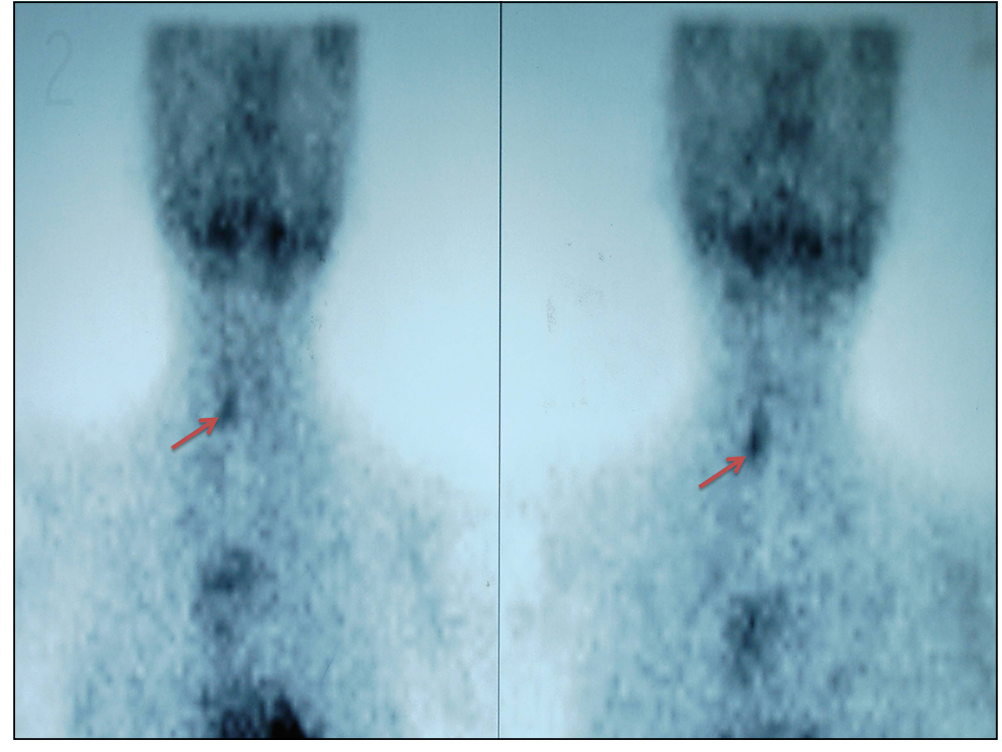

Figura 4. Gammagrafía con Sestamibi que demuestra una zona de hipercaptación paratiroidea derecha (Flecha roja).

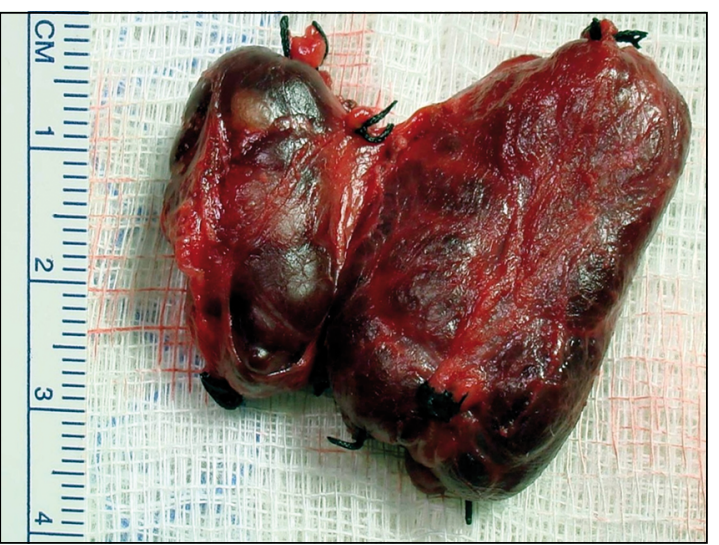

Figura 5. Resección multiestructural de tumor paratiroideo y lóbulo tiroideo derecho.

Existe un predominio del hiperparatiroidismo primario en las mujeres con una relación $3: 1^{10}$, y su prevalencia aumenta con la edad de $0,5 \%$ en mujeres de 50 a 59 años a $1,75 \%$ en mujeres de 70 a 75 años $^{12}$. En países occidentales la sintomatología del hiperparatiroidismo primario es muy leve y su progresión es muy lenta ${ }^{13}$.

Los síntomas y signos clásicos del hiperparatiroidismo primario son: dolor articular, litiasis renal, manifestaciones abdominales y psíquicas ${ }^{14}$; pero con la investigación de los niveles séricos de calcio en los chequeos de rutina, el número de casos asintomáticos ha ido en aumento y los casos sintomáticos

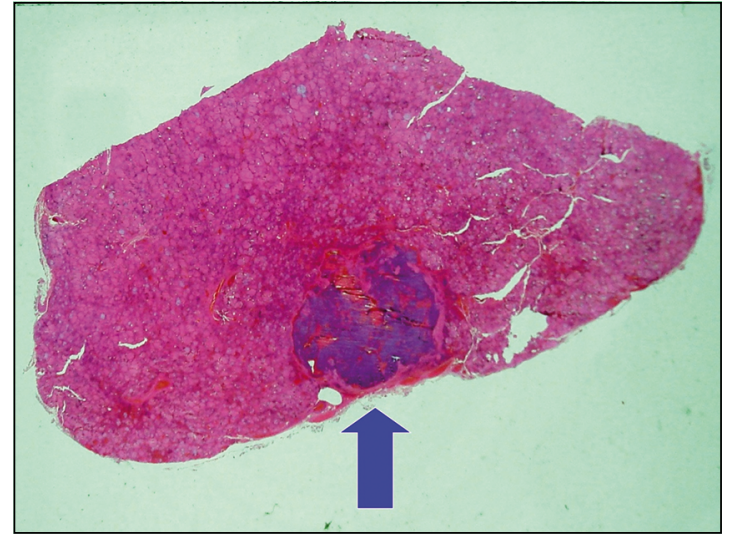

Figura 6. Histopatología que demuestra un micro carcinoma papilar de tiroides en el lóbulo derecho.

constituyen la minoría ${ }^{15}$. Actualmente, la Gammagrafía con Sestamibi-Tc99m se considera como una herramienta con mayor sensibilidad (80-100\%) para detectar hiperfunción paratiroidea y glándulas ectópicas ${ }^{16}$.

Las complicaciones esqueléticas del hiperparatiroidismo primario (osteítis fibrosa quística, tumores pardos, quistes óseos, fracturas y deformidades) ${ }^{6}$ se presentan en menos del 5\% de los pacientes y la incidencia de litiasis renal ha disminuido en un 15 al $20 \%{ }^{17}$, debido a la detección temprana de esta patología.

La mayoría de tumores de células gigantes son 
asintomáticos, pero la principal manifestación es el dolor óseo por efecto local del crecimiento tumoral y su tratamiento es esencialmente quirúrgico ${ }^{18,19}$. Muchas de las características radiológicas del tumor pardo son similares a las de un tumor de células gigantes del hueso y no existen datos histológicos patognomónicos para el diagnóstico de tumor pardo, por lo que es muy difícil de distinguir estas dos pato$\operatorname{logías}^{20}$, en nuestro caso el servicio de traumatología realizó una resección ósea que es el tratamiento específico para el tumor de células gigantes.

El Tumor Pardo no es una verdadera neoplasia ${ }^{21}$ y se atribuye a Gerhard Engel en 1864 su primera descripción, y el reconocimiento de su asociación con el hiperparatiroidismo en 1925 por $\mathrm{Mandl}^{22}$, este se presenta en el $4,5 \%$ de los pacientes con hiperparatiroidismo primario y en el 1,5 al $1,7 \%$ del secundario ${ }^{23}$. Es producido por el incremento de la actividad osteoclástica donde el tejido óseo normal es reemplazado por tejido fibroso vascular y de granulación que da como resultado la formación de células gigantes y su posterior expansión ${ }^{7,24}$; estas lesiones pueden aparecer en cualquier parte del esqueleto y afectar a uno o más huesos ${ }^{25}$.

Clínicamente se manifiestan por fracturas asociado a la variedad de síntomas del hiperparatiroidismo ${ }^{26}$. Radiográficamente estos tumores se presentan como lesiones líticas uni o multiloculares bien definidas con escasa formación ósea ${ }^{26,27}$. En la Resonancia Magnética Nuclear estas lesiones son hipointensas en la secuencia $\mathrm{T} 1 \mathrm{y}$ de densidad mixta en la secuencia T2, esto debido a la susceptibilidad magnética de la hemosiderina, la matriz osteoide y aire 27,28

Las características histológicas de estos tumores son la presencia de aumento en el número de osteoclastos y osteoblastos, el tejido óseo trabecular se encuentra engrosado y rodeado por tejido fibroso, células gigantes, células inflamatorias y depósitos de hemosiderina ${ }^{29}$, características que son similares al tumor de células gigantes, por lo que su diagnóstico diferencial se realiza en base a la clínica y datos bioquímicos como son hipercalcemia y niveles elevados de paratohormona ${ }^{20}$.

El tratamiento primario de los tumores pardos es la extirpación quirúrgica de la glándula paratiroidea patológica, con este tratamiento la mayoría de los tumores involucionan en un tiempo de 6 meses a 5 años ${ }^{30}$; los niveles séricos normales de paratohormona, calcio, fósforo y el aumento de la masa ósea en el área tumoral son indicadores de éxito del tratamiento ${ }^{31}$. La resección quirúrgica ósea está indicada en lesiones quísticas extensas con gran destrucción ósea que no involucionan una vez corregida la causa del hiperparatiroidismo ${ }^{30,32}$.

La asociación de un micro carcinoma papilar ti- roideo con el adenoma paratiroideo fue descrita por primera vez en 1956 por Ogburn y colaboradores ${ }^{34}$, con una frecuencia que oscila entre el $3,1-17 \%$ según los diferentes reportes ${ }^{35}$.

No existe una clara asociación entre estas dos patologías, se han documentado posibles etiologías como el efecto oncogénico de la hipercalcemia ${ }^{36}$, el incremento del factor de crecimiento epitelial y el factor de crecimiento similar a la insulina ${ }^{34,37}$, bajas dosis de radiación en cabeza y cuello en la infancia que pueden relacionarse con la sincronía de tumores tiroideos y paratiroideos ${ }^{38}$.

A pesar de que la coexistencia de estas dos entidades es rara, el diagnóstico de patología tiroidea debe ser considerado en aquellos pacientes con hiperparatiroidismo primario, para su resolución simultánea ${ }^{40}$.

\section{Conclusiones}

El presente estudio permite determinar que el Hiperparatiroidismo Primario grave puede simular un Carcinoma Paratiroideo con metástasis óseas.

El hiperparatiroidismo puede conllevar a la formación de pseudo tumores óseos conocidos como Tumores Pardos producidos por la intensa actividad osteoclástica.

El diagnóstico diferencial del Tumor Pardo y el Tumor de células Gigantes no se puede realizar sólo mediante el estudio de histopatología, se debe evaluar los datos clínicos y bioquímicos del paciente.

Este caso nos permite corroborar la asociación entre hipercalcemia y el desarrollo de Carcinoma Tiroideo, por lo tanto, se sugiere realizar estudios complementarios de la glándula tiroides en aquellos pacientes con hiperparatiroidismo para proporcionar un tratamiento quirúrgico adecuado.

\section{Referencias}

1. MacKenzie-Feder J, Sirrs S, Anderson D, Khan A. Primary Hyperparathyroidism: An Overview. Int J Endocrinol. 2011;2011:251410.

2. Bilezikian J. Primary hyperparathyroidism. Updated March 7, 2011 (http://www.endotext.org/parathyroid/ parathyroid5/parathyroidframe5.htm).

3. Wermers RA, Khosla S, Atkinson EJ, Achenbach SJ, Oberg AL, Grant CS, et al. Incidence of primary hyperparathyroidism in Rochester, Minnesota, 1993-2001: an update on the changing epidemiology of the disease. J Bone Miner Res. 2006;21:171-7.

4. Sitges-Serra A, Bergenfelz A. Clinical update: Sporadic primary hyperparathyroidism. Lancet 2007;370:46870 . 
5. Ayuk J, Cooper M, Gittoes N. New perspectives in the management of primary hyperparathyroidism. Ther Adv Endocrinol Metab. 2010;5:197- 205.

6. Marcocci C, Cianferotti L, Cetani F. Bone disease in primary hyperparathyrodism. Ther Adv Musculoskel Dis. 2012;4:357- 68.

7. Mantar F, Gunduz S, Gunduz U. A Reference Finding Rarely Seen in Primary Hyperparathyroidism: Brown Tumor. Hindawi Publishing Corporation Case Reports in Medicine Volume 2012, Article ID 432676, 4 pages.

8. Hellwig C. Thyroid adenoma in experimental animals. Amer J Cancer 1935;23:550- 5.

9. Taylor S. Calcium as a goitrogen. J. Clin. Endoer. 1954;14:1412-22.

10. Fraser W. Hyperparathyroidism. Lancet 2009;374:14558.

11. Kruse A, Beige J. Hyperparathyroidism-new aspects. Ther Umsch. 2007;64:265- 9.

12. Jorde R, Bonaa K, Sundsfjord J. Primary hyperparathyroidism detected in a health screening. The Tromso study. Journal of Clinical Epidemiology 2000;53:11649.

13. Mihai R, Wass J, Sadler G. Asymptomatic hyperparathyroidism-need for multicentre studies. Clinical Endocrinology 2008;68:155-64.

14. Kaplan E, Yashiro T, Salti G. Primary hyperparathyroidism in the 1990s: choice of surgical procedures for this disease. Annals of Surgery 1992;215:300-17.

15. Bilezikian J, Silverberg S. Asymptomatic primary hyperparathyroidism. The New England Journal of Medicine 2004;350:1746-51.

16. Javadi H, Jallalat S, Farrokhi S, Semnani S, Mogharrabi M, Riazi A, et al. Concurrent papillary thyroid cancer and parathyroid adenoma as a rare condition: a case report. Nuclear Med Rev. 2012;15:153-5.

17. Silverberg S, Shane E, Jacobs T, Siris E, Gartenberg F, Seldin D, et al. Nephrolithiasis and bone involvement in primary hyperparathyroidism. Am J Med. 1990;89:32734.

18. Lau Y, Sabokbar A, Gibbons C, Giele H, Athanasou N. Phenotypic and molecular studies on giant cell tumours of bone and soft tissues. Hum Pathol. 2005;36:945- 54.

19. Balke M, Campanacci L, Gebert C, Picci P, Gibbons M, Taylor R, et al. Bisphosphonate treatment of aggressive primary, recurrent and metastatic giant cell tumour of bone. Cancer 2010;10: 462. doi: 10.1186/1471- 240710- 462 .

20. Vera L, Dolcino M, Mora M, Oddo S, Gualco M, Minuto F, et al. Primary hyperparathyroidism diagnosed after surgical ablation of a costal mass mistaken for giant-cell bone tumor: a case report. J Med Case Rep. 2011;28;5:596. doi: 10.1186/1752-1947-5-596.

21. Ullah E, Ahmad M, Asmat Ali, Redhu N. Primary hyperparathyroidism having multiple Brown tumors mimicking malignancy. Indian J Endocr Metab. 2012;16:1040- 2.
22. Buchanan W, Kraag G, Palmer D, Cockshott WP . The first recorded case of osteitis fibrosa cystica. Can Med Assoc J. 1981;124:812-5.

23. Keyser J, Postma G. Brown tumor of the mandible. Am J Otolaryngol. 1996;17:407-10.

24. Campuzano G, Pérez W, Marín J. A 60-year-old man with chronic renal failure and a costal mass: a case report and review of the literature. J Med Case Rep. 2009;3:7285.

25. Pinto M, Sass S, Sampaio C, Campos D. Brown tumor in a patient with hyperparathyroidism secondary to chronic renal failure. Braz J Otorhinolaryngol. 2010;76:404.

26. Zhang H. Multiple Brown Tumors. A Diagnostic Dilemma. The Endocrinologist 2010;20:152-4.

27. Takeshita T, Takeshita K, Abe S, Takami H, Imamura T, Furui S. Brown tumor with fluid- fluid levels in a patient with primary hyperparathyroidism: Radiological finding. Radiat Med. 2006;24:631- 4.

28. Knowles N, Simith D, Outwater E. MRI diagnosis of brown tumor based on magnetic susceptibility. J Magn Reson Imaging 2008;28:759-61.

29. Khalil P, Heining S, Huss R, Ihrler S, Siebeck M, Hallfeldt $\mathrm{K}$, et al. Natural history and surgical treatment of brown tumor lesions at various sites in refractory primary hyperparathyroidism. Eur J Med Res. 2007;12:222-30.

30. Chavin H, Pisarevsky A, Chavin C, Koll L, Petrucci E. Tumor pardo como manifestación inicial del hiperparatiroidismo primario. Medicina 2008;68:219-21.

31. Moreira C, Bandeira C, Voss D. Marked improvement in bone mass after parathyroidectomy in osteitis fibrosa cystic. Journal of Clinical Endocrinology and Metabolism 1998;83:732-5.

32. Yamazaki H, Ota Y, Aoki T, Karakira H. Brown Tumor of the maxilla and mandibule: progressive mandibular brown tumor after removal of parathyroid adenoma. J Oral Maxillofac Surg. 2003;61:719-23.

33. Shane E. Clinical review: Parathyroid carcinoma. J Clin Endocrinol Metab. 2001;86:485-93.

34. Ogburn P, Black B. Primary hyperparathyroidism and papillary adenocarcinoma of the thyroid: report of four cases. Proc Staff Meet Mayo Clin. 1956;31:295- 8.

35. dell'Erba L, Baldari S, Borsato N, Bruno G, CalòGabrieli G, Carletto M. Retrospective analysis of the association of nodular goiter with primary and secondary hyperparathyroidism. Eur J Endocrinol. 2001;145:42934.

36. Ho K. Papillary parathyroid adenoma. A rare occurrence and its importance in differentiation from papillary carcinoma of the thyroid. Arch Pathol Lab Med. 1996;120:883-4.

37. McCarty M. Parathyroid hormone may be a cancer promoter-an explanation for the decrease in cancer risk associated with ultraviolet light, calcium, and vitamin D. Med Hypotheses 2000;54:475-82. 
38. Stephen A, Chen K, Milas M, Siperstein A. The coming of age of radiation-induced hyperparathyroidism: evolving patterns of thyroid and parathyroid disease after head and neck irradiation. Surgery 2004;136:114353.

39. Javadi H, Jallalat S, Farrokhi S, Semnani S, Mogharrabi M, Riazi A, et al. Concurrent papillary thyroid cancer and parathyroid adenoma as a rare condition: a case report. Nuclear Med Rev. 2012;15:153-5.

40. Siperstein A, Berber E, Mackey R, Alghoul M, Wagner K, Milas M. Prospective evaluation of sestamibi scan, ultrasonography, and rapid PTH to predict the success of limited exploration for sporadic primary hyperparathyroidism. Surgery 2004;136:872- 80 . 